\title{
Optimizing COVID-19 Health Campaigns: A Person-Centered Approach
}

\author{
Rachel A. Smith ${ }^{\mathrm{a}^{*}}$, Jessica G. Myrick ${ }^{\mathrm{b}}$, Robert P. Lennon ${ }^{\mathrm{c}}$, Molly A. Martin ${ }^{\mathrm{d}}$, Meg L. Smalle, \\ Lauren J. Van Scoy ${ }^{\mathrm{f}}$, and the Data4Action Research Group
}

${ }^{a}$ Department of Communication Arts and Sciences, Pennsylvania State University, University Park, PA, USA ${ }^{b}$ Donald P. Bellisario College of Communications, Pennsylvania State University, University Park, PA, USA; ' Department of Family and Community Medicine, Pennsylvania State College of Medicine, Hershey, PA, USA. ORCID ID: 0000-0003-0973-5890; ${ }^{d}$ Department of Sociology and Criminology, Pennsylvania State University, University Park, PA, USA; ${ }^{e}$ College of Health and Human Development, Pennsylvania State University, University Park, PA, USA; ${ }^{f}$ Department of Medicine, Pennsylvania State College of Medicine, Hershey, Pennsylvania.

Corresponding author Rachel A. Smith, 234 Sparks Building, University Park, PA 16802, USA. Tele: 814.865.4201. Email: ras57@psu.edu

Acknowledgements: The authors thank the members of the D4A Action Research Group: Dee Bagshaw, Clinical \& Translational Science Institute, Nita Bharti, Dept. of Biology and the Huck Institutes of the Life Sciences, Cyndi Flanagan, Clinical Research Center, Matthew Ferrari, Dept. of Biology \& Huck Institutes of the Life Sciences, Thomas Gates, Social Science Research Institute, Margeaux Gray, Dept. of Biobehavioral Health, Suresh Kuchipudi, Animal Diagnostic Lab, Vivek Kapur, Dept. of Animal Science and the Huck Institutes of Life Sciences, Stephanie Lanza, Dept. of Biobehavioral Health and the Prevention Research Center, James Marden, Dept. of Biology \& Huck Institutes of the Life Sciences, Susan McHale, Dept. of Human Development and Family Studies and the Social Science Research Institute, Glenda Palmer, Social Science 
Research Institute, Andrew Read, Depts. of Biology and Entomology, and the Huck Institutes of the Life Sciences, Connie Rogers, Dept. of Nutritional Sciences and the Huck Institutes of the Life Sciences, and Charima Young, The Penn State Office of Government and Community Relations.

Declaration of interest statement: The authors report no conflicts of interest. Members of the Data4Action Research Group include leaders in each funding source. Those leaders participated in developing the project concept and methodologies and study design. They were not involved in analysis, interpretation of the data, initial writing, or the decision to submit the report for publication. 


\begin{abstract}
As the United States continues to be ravaged by COVID-19, it becomes increasingly important to implement effective public health campaigns to improve personal behaviors that help control the spread of the virus. To design effective campaigns, research is needed to understand the current mitigation intentions of the general public, diversity in those intentions, and theoretical predictors of them. COVID-19 campaigns will be particularly challenging because mitigation involves myriad, diverse behaviors. This study takes a person-centered approach to investigate data from a survey $(N=976)$ of Pennsylvania adults. Latent class analysis revealed five classes of mitigation: one marked by complete adherence with health recommendations $(34 \%$ of the sample), one by complete refusal ( $9 \%$ of the sample), and three by a mixture of adherence and refusal. Statistically significant covariates of class membership included relatively negative injunctive norms, risk due to essential workers in the household, personal knowledge of someone who became infected with COVID-19, and belief that COVID-19 was a leaked biological weapon. Additionally, trait reactance was associated with non-adherence while health mavenism was associated with adherence. These findings may be used to good effect by local healthcare providers and institutions, and also inform broader policy-making decisions regarding public health campaigns to mitigate COVID-19.
\end{abstract}

Keywords: COVID-19, mitigation, injunctive norms, risk, audience analysis, latent class analysis 


\section{Optimizing COVID-19 Health Campaigns: A Person-Centered Approach}

In early 2020, the WHO characterized a novel coronavirus, SARS-CoV-2, as a public health emergency and, about six weeks later, declared the COVID-19 pandemic (Cucinotta \& Vanelli, 2020). The WHO encouraged personal mitigation efforts as well as institutional and national efforts to handle diagnosis, containment, and clinical management. These efforts were, in part, to buy time while the world raced to create a coronavirus vaccine. Even with a vaccine, though, mitigation efforts may need to be sustained for some time, particularly if vaccine distribution or uptake are slow.

Because of this need to continue mitigation efforts, it is crucial to remember that the general public can play a vital role in slowing the transmission rate of COVID-19 through personal behaviors. The U.S. Centers for Disease Control and Prevention (CDC, 2020a) identified three broad categories of prevention behaviors: hygiene (e.g., wash your hands often), face masks, and social distancing. The CDC also noted actions to manage symptoms: covering the nose or mouth if a person coughs or sneezes, staying home, and seeking medical attention when ill or potentially exposed. Given these behaviors have been politically polarized in the U.S. (Alsan et al., 2020), highly effective public health campaigns are needed to encourage their widespread adoption.

Effective campaigns provide a target audience with the right persuasive strategy to inspire change based on their initial state and psychosocial predictors for change (Maibach et al., 1996; Slater, 1996; Smith, 2017). A challenging problem for health campaigns is defining those who need to be reached, and then intelligently grouping people based on a complex set of variables in order to identify audiences with similar needs who will respond well to a particular persuasive strategy. Audience segmentation must be a systematic and explicit research process 
involving informed decisions about how many subgroups of audiences to consider, why the subgroups do what they do, and how to reach each subgroup effectively. Early studies evaluated intent to comply and actual compliance with CDC recommendations and found widespread differences by region, race, and education level (e.g., Block et al., 2020; Lennon et al., 2020). Others show that COVID-19 knowledge and perceptions are related more to education level alone than by race or gender (Van Scoy et al., in press). This suggests that effective health campaigns will need to look beyond basic demographics, and, instead, segment people into groups based on their COVID-19-specific behaviors.

COVID-19 mitigation presents another challenge to current theories and methods for campaign design and audience segmentation because mitigation involves many, diverse behaviors. The present study takes a person-centered approach to investigate the patterns of behavioral intentions of adults residing in a mid-Atlantic county that includes a large university. While person-centered methods enable informed decisions about with whom to intervene, supporting theory is needed to understand how to do so effectively (Maibach et al., 1996; Maibach et al., 2011; Slater, 1996; Slater et al., 2006; Smith, 2017). To that end, we include a covariate analysis of theoretically relevant predictors to anticipate strategic communication efforts.

\section{Campaign Design for Complex Mitigation}

Audience segmentation provides critical information for planning effective campaigns that use resources strategically (Maibach et al., 2011; Smith, 2017). No two people are exactly alike with respect to their risk for illness or their reactions to a campaign attempting to lower their risk. It is often financially and logistically unreasonable to create campaigns for each individual within a population; it is even unnecessary to the extent to which people exist in 
similar states and share psychosocial predictors for change. The prevailing guidance encourages campaign designers to identify audience segments based on shared determinants of a behavior (Slater, 1996). Indeed, most theories of behavior and behavior change, like the theory of reasoned action (Fishbein \& Ajzen, 2010), limit their scope to one behavior at a time and its unique psychosocial determinants. How should we approach campaign planning when the public needs to engage in multiple behaviors, such as with COVID-19 mitigation?

One option, under the prevailing guidance, is to target each behavior associated with COVID-19 mitigation separately and identify its determinants. Targeting particular behaviors presumes that acts of mitigation are not interrelated and assumes that mitigation patterns are not determined by a common cause. Both assumptions may not be plausible for every health threat. A second option is to assume that acts of mitigation have a single latent-factor structure: handwashing, mask-wearing, and social distancing are all manifestations of a single intention to limit the spread of COVID-19. This structure, however, is not typically supported by evidence: people often exhibit complex patterns of compliance and non-compliance (Bochner \& Insko, 1966; Smith et al., 2018), including with COVID-19 recommendations (Lennon et al., 2020).

An alternative strategy is to explore audience segmentation through person-centered methods (for a review see Smith, 2017) to identify subgroups based on shared, targeted behaviors - in this case, intentions to engage in COVID-19 mitigation. One popular technique is latent class analysis (LCA; Collins \& Lanza, 2010). LCA attempts to capture latent constructs from measurable variables when the latent construct is categorical (Collins \& Lanza, 2010), such as latent subgroups of adults with similar forms of COVID-19 mitigation. This focus on behaviors aligns with a lifestyle analysis (Slater \& Flora, 1991), which allows one to understand how many behaviors may need to change within a given segment. Some behavioral profiles 
could be identified as more problematic for public health (by number of problematic behaviors or greater severity of consequences from the behavior), which could help to prioritize which segments to target first when resources are limited. In addition, there may also be "low-hanging fruit;" behaviors within certain profiles for which low cost interventions could yield large health gains. Assuming that profiles with more problem behaviors will be more difficult to change, researchers can anticipate which segments may need stronger persuasive appeals.

Research Question 1: Is there a latent class structure that adequately represents the heterogeneity in intentions to engage in COVID-19 mitigation?

\section{Predicting Profiles of COVID-19 Mitigation}

One purpose of segmenting an audience into subgroups is to make communication efforts more effective and efficient (Slater, 1996). However, the most efficient campaign possible—one, general message for everyone - is less effective in gaining behavior change than crafting messages targeting groups of people or tailoring them to a particular individual (Noar et al., 2007). Targeted messages are more likely to be read, understood, recalled, perceived as credible, and rated highly than one, generalized communication (e.g., Kreuter et al., 2000; Noar et al., 2007). To develop theoretically guided messages for subgroups (Slater \& Flora, 1991; Storey et al., 2008), we turn to existing models of behavior and behavior change and explore predictor variables associated with both adherence and non-adherence.

Norms

A key predictor of health behavior is perceived norms (Sheeran et al, 2016): what people believe is typical, accepted, and required by others. Two types of norms (Cialdini \& Goldstein, 2004; Rimal \& Lapinski, 2015) are descriptive norms (what most people are doing) and injunctive norms (what the people important to me want me to do). According to the theory of 
normative social behavior (Rimal \& Real, 2005), descriptive norms are most relevant when predicting new, unfamiliar, or uncertain behaviors. Thus, descriptive norms may be quite useful to predict the early days of COVID-19 vaccination. The length of this pandemic has provided the opportunity to investigate mitigation behaviors at different phases of the outbreak. At the time of this data collection (August-mid October, 2020), U.S residents had been enacting COVID-related health behaviors for months. The uncertainty, novelty, and unfamiliarity likely had worn off. However, injunctive norms could still be quite relevant to these behaviors.

Injunctive norms refer to people's belief in the social approval or disapproval of their behavior by important others (Park \& Smith, 2007; Reno et al., 1993). The mechanism of influence is based in affiliation and conformity (Cialdini \& Goldstein, 2004; Rimal \& Lapinski, 2015): if we engage in behaviors that others approve and avoid behaviors that others disapprove, then we can maintain and sustain our social relationships. Current research (Goldberg et al., 2020) shows that injunctive norms predict COVID-19 mitigation behaviors; this study, as seen with most studies of normative influence, focused entirely on approval. And yet, disapproval is at the heart of conformity: people actively, consciously, and deliberately attempt to gain others' approval by conforming, especially when they experience or even witness disapproval or rejection for their behavior (Cialdini \& Goldstein, 2004). We predict:

Hypothesis 1: Experiencing more negative than positive injunctive norms predicts lower intentions to engage in COVID-19 mitigation.

Risk

Multiple theories (extended parallel process model, Witte, 1994; health belief model, Becker, 1974; protection motivation theory, Rogers, 1975) include risk as a key predictor of health behavior. The hazard outrage model of risk communication (HOM; Sandman, 1993) 
explains that the public makes sense of risk based on the hazard, which is the assessment of technical concern (severity and vulnerability), and outrage, which is the assessment of cultural or social concern (e.g., fairness, morality, natural vs man-made). For example, for COVID-19, being an essential worker increases the possibility of being exposed to the virus, and having chronic disease increases the likelihood of experiencing more serious symptoms (CDC, 2020b). Knowing someone who was infected with COVID-19 can also shape perceptions of the magnitude and the probability of being infected (for examples with cancer risk perceptions see Montgomery et al., 2003) given our social networks generally comprise people very similar to ourselves (McPherson et al., 2001). These examples emphasize hazard aspects of risk: severity of and susceptibility to the health risk.

One source of outrage is whether the risk is framed as natural or man-made (Sandman, 1993): man-made threats generate more outrage than natural threats and focus the public's behavioral actions on the source of the threat (e.g., yelling at people), which may have little to do with recommended mitigation (e.g., handwashing). In the case of COVID-19, one myth that spread through some segments of the public is that COVID-19 was man-made and leaked from a biological weapons laboratory (Aiyewumi \& Okeke, 2020). This belief focuses the risk on a (mythic) villain and retaliation, which coincides with the marked increase in anti-Asian hate crimes during the pandemic (Gover et al., 2020); but, is unlikely to lead to an increase in handwashing. Sandman (1993) notes that experts and the public often differ in their focus "the overarching problem is that the public cares too little about the hazard, and the experts care too little about the outrage" (p. 8). According to the HOM, although both hazard and outrage can motivate the public to engage in behavior, outrage is the stronger predictor of public engagement. We predict: 
Hypothesis 2: Greater vulnerability due to more essential workers in one's household or more household members with existing chronic disease predicts higher intentions to engage in COVID-19 mitigation.

Hypothesis 3: Knowing someone (vs. no one) who became sick with COVID-19 predicts higher intentions to engage in COVID-19 mitigation.

Hypothesis 4: Stronger bioweapon belief that COVID-19 was a man-made, leaked biological weapon predicts lower intentions to engage in COVID-19 mitigation.

\section{Reactance and Health Mavenism}

In addition to norms and risk, which may be modifiable via campaigns, it is also important to identify behavioral patterns of people with traits known to shape message processing and with attributes of opinion leaders. As such, we considered trait reactance and the opinion leader trait of health mavenism.

As people feel that their freedom is threatened, they experience reactance, which motivates them to restore their threatened freedom by counterarguing or engaging in the forbidden act (Brehm \& Brehm, 1981; Dillard \& Shen, 2005). The state of reactance can be caused by situational factors. For example, during the course of COVID-19, some states and localities in the U.S. have mandated wearing face masks in public places and avoiding crowds. People also vary in "their trait proneness to reactance arousal" (Dillard \& Shen, 2005, p. 149). Although any persuasive message can be perceived as a threat to one's freedom, mandates inherently create a loss of freedom. On the other hand, reviews of reactance research indicate that sometimes "a person will give up a freedom when it is clear there is no way to recover it" (Burgoon et al., 2002, p. 222). Thus, it is important to identify whether people with stronger trait 
reactance show a particular profile of mitigation, and then craft messages for them without freedom-threatening language.

Some people serve as opinion leaders for others in their social networks. Boster and colleagues (2011) described three different personality traits of opinion leaders: mavenism, persuasiveness, and connectivity. This study focuses on health mavens. Health mavens actively maintain expertise in a variety of areas, share their knowledge, and are likely to be resources for others (Boster et al., 2011). Boster and colleagues (2011) argued that identifying people with these qualities may be useful to efforts to diffuse health information via opinion leadership. They also may be the source of counter-messages in a community. We considered:

Research Question 2: Do (a) trait reactance and (b) health mavenism predict membership in COVID-19 mitigation latent classes?

In summary, we build on prior theoretical and empirical research to question whether and how people's perceptions of injunctive norms and risks, as well as their personal levels of trait reactance and health mavenism, predict the patterning of their engagement in COVID-19 mitigation strategies. This timely research can provide information to craft more effective and efficient health messaging strategies and potentially curb the spread of COVID- 19.

\section{Methods}

\section{Participants}

Participants $(N=976)$, on average, were 49 years old $(M=49.27, S D=16.26$, Median $=$ 53, $\operatorname{Min}=18, \operatorname{Max}=90)$. Participants self-identified as male $(31 \%)$, female $(67 \%)$, transgender male $(.2 \%)$, transgender female $(.1 \%)$, non-binary $(.8 \%)$, or did not answer $(.8 \%)$. Participants self-identified as heterosexual (89\%), gay or lesbian (4\%), bisexual (4\%), queer (.5), asexual $(.4 \%)$, or did not answer (2\%). Participants self-identified, selecting as many choices as they 
liked, as Caucasian (93\%), Asian (4\%), African American (1\%), and American Indian or Alaska Native (.4\%), or did not answer (2\%); $5 \%$ identified as Hispanic. Participants reported the following occupations: employed (74\%), unemployed (2\%), retired (18\%), homemaker (2\%), student (9\%), and active duty military $(.1 \%) ; 2 \%$ were receiving unemployment benefits. On average, participants perceived their financial standing (Adler \& Stewart, 2007) as good ( $M=$ 7.04, $S D=1.50$, Median $=7.00$, Min = 1, Max = 10). Participants' education varied: $2 \%$ had graduated from high school, $7 \%$ had some college experience, $5 \%$ had graduated with an associate's degree, 29\% had graduated with an undergraduate degree, and 57\% had completed graduate education.

\section{Design and Procedures}

This is a cross-sectional study from time 1 of a prospective, longitudinal cohort living in one county in a mid-Atlantic state; the county has a large university in it. This study was approved by an institutional review board. Participants were recruited between May and September 2020 through employers, local government, news coverage, and word-of-mouth. Inclusion criteria were (a) age 18 years or older, (b) residence in the county of interest with a continuous physical presence since March 2020, and (c) without co-residential children under age 18 (caregivers received a different survey). Data were collected between August and midOctober, 2020. The data presented in this analysis come from a larger project, including other surveys and clinical data. Of the total sample, 1009 were invited to complete this survey, and 976 answered the items used in this analysis ( $97 \%$ completion rate).

\section{Latent Class Indicators}

Eight items were used to assess participants' intentions to engage in recommended actions related to COVID-19. Responses were marked on 5-point scales $(1=$ never to $5=$ 
always). The responses were collapsed into two categories for the LCA: never, rarely, sometimes, and most of the time, were separated from always. This coding emphasizes the goal of persuading people to intend to engage in the recommended actions; those who were uncertain would be targeted to move toward intending to engage in the recommended behavior all the time. Table 1 shows the codes for each item.

\section{Covariates}

Relatively Negative Injunctive Norms

Three items were used to assess negative injunctive norms (e.g., "They make fun of me for wearing a face covering;" $\alpha=.78$ ); three other items were used to assess positive injunctive norms $(\alpha=.77)$. Responses, marked on 5 -point scales $(1=$ strongly disagree, $5=$ strongly agree), were averaged into separate scores. Positive injunctive norms was subtracted from negative injunctive norms, thus higher scores indicate relatively more negative (than positive) injunctive norms $(M=-3.34, S D=1.07, \operatorname{Min}=-4, \operatorname{Max}=2.67)$.

Household Risk: Essential Worker and Chronic Disease

Participants were asked if they or others in their household were (a) at increased risk for exposure to COVID-19 due to their employment, or (b) had a chronic medical condition. Responses included me, spouse, children, other household members, or no one and were summed into continuous scores $(0=$ no one, $4=$ checked all household options; $M=0.44, S D=$ $0.68, \operatorname{Min}=0, \operatorname{Max}=4$ for essential worker risk and $M=0.48, S D=0.68, \operatorname{Min}=0, \operatorname{Max}=4$ for chronic disease risk).

Personal Knowledge 
Participants were asked if they personally knew someone who has gotten infected with COVID-19 outside of their household. Responses were marked as yes $(1 ; 57 \%$ of the sample) or no $(0 ; 43 \%$ of the sample).

\section{Biological Weapon Belief}

Two items were used to assess belief that COVID-19 was a man-made, biological weapon (e.g., "I think that COVID-19 is a biological weapon that leaked from a laboratory"). Responses, marked on 5-point scales ( 1 = strongly disagree, 5 = strongly agree $)$, were averaged into one score $(r=.72, p<.001)$; higher scores indicate stronger belief.

\section{Trait Reactance}

Five items from the Hong psychological reactance scale (Hong \& Faedda, 1996; subscales of resisting influence and reactance to recommendations, Shen \& Dillard, 2005) were used to assess trait psychological reactance (e.g., "When someone forces me to do something, I feel like doing the opposite"). Responses, marked on 5-point scales $(1=$ strongly disagree to $5=$ strongly agree), were averaged into one score $(\alpha=.73)$; higher scores indicate stronger trait reactance.

\section{Health Mavenism}

Four items adapted from Boster et al. (2011) were used to assess identification as a health maven (e.g., "I like to be aware of the most up-to-date healthy lifestyle information so I can help others by sharing it' '). Responses, marked on 5-point scales ( $1=$ strongly disagree, $5=$ strongly agree), were averaged into one score $(\alpha=.84)$; higher scores indicate stronger mavenism.

\section{Results}

\section{Descriptive Statistics}


As seen in Table 1, most participants intend to stay home if they felt sick (86\%), seek medical attention if they became symptomatic (84\%), cover their mouth or nose when they cough or sneeze $(80 \%)$, and wear a face mask when out in public $(77 \%)$. Fewer intend to avoid crowded locations and mass gatherings (65\%), and about half intend to wash their hands regularly with soap (52\%) and stay six feet away from other people when they are in public settings (49\%). Fewer than half intend to avoid touching their faces with unwashed hands (40\%).

\section{Latent Class Analysis}

To address RQ1, PROC LCA (Lanza et al., 2007) was used in SAS 9.4 to calculate the fit indices for two- to seven-class models (see Table 2). Each test was run using 100 random starting values. PROC LCA provides goodness-of-fit indicators for models, which were used to select the best number of classes (e.g., a four- or five-class model; Collins \& Lanza, 2010). Good models should have a goodness-of-fit statistic ( $G^{2}$ value) less than the degrees of freedom (Collins \& Lanza, 2010). The two-, three-, and four-class models did not fit this criterion. Akaike information criterion (AIC) and Bayesian information criterion (BIC) estimates are used to select the best model; lower scores are better. The AIC estimates minimized at the six-class model, and the BIC estimates minimized at the five-class model. This disparity is not uncommon (Collins \& Lanza, 2010). We selected the five-class model because the BIC estimating procedure emphasizes parsimony (Collins \& Lanza, 2010) and the difference in the five- and six-class models was to split the smallest classes into two even smaller classes with similar profiles.

Table 3 shows the item-response probabilities for the five-class model and the likelihood of reporting a behavior (i.e., the responses that were coded as 2 in Table 1) within a class. The largest group (34\%), labeled Adherents, had very high likelihoods of intending to engage in all recommended behaviors. In contrast, the smallest group (9\%), labeled Refusers, had very low 
likelihoods of intending to engage in any of the recommended behaviors. The other three groups differed in their patterns of intentions. One group (10\%), labeled Symptom Managers, had high likelihoods of intending to stay home if they were ill and to seek medical attention if they became symptomatic, and a moderate likelihood of intending to cover their face as they cough or sneeze, but had low likelihoods of intending to engage in any of the other recommended behaviors. Another group (23\%), labeled Hygiene Stewards, also had a high likelihood of attending to symptoms and also intended to wash their hands and to wear a face mask, but nothing else. The last group (24\%), labeled Social Distancers, had a high likelihood of attending to symptoms, and also intended to wear a face mask, avoid crowds, and probably keep six feet of physical distance from other people, but nothing else.

\section{Covariate Analysis}

H1-3 and RQ1 explored whether relatively negative injunctive norms, risks due to essential workers or chronic disease in the household, personal knowledge of someone who became infected with COVID-19, belief that COVID-19 was a leaked biological weapon, trait reactance, or health mavenism predicted membership in the classes of COVID-19 mitigation. Of note, the correlations between these predictors were small (-.07 to .17$)$. Table 4 shows the results of this analysis, with Adherent as the referent class. All of the predictors, except for chronic disease risk, were statistically significant covariates of class membership at $p<.05$.

In comparison to Adherent, participants who reported experiencing more negative than positive injunctive norms had higher odds of being in all of the other problematic classes, supporting H1. Participants who reported more essential workers in their household had higher odds of being Hygiene Stewards, Symptom Managers, and Refusers, in comparison to Adherent, but lower odds of being Social Distancers, which contradicts H2a. The number of household 
members with chronic disease did not predict class membership, which does not support H2b. Participants who knew someone outside of their household who had gotten sick with COVID-19 had higher odds of being Symptom Managers and Refusers, in comparison to Adherent, but lower odds of being Social Distancers and Hygiene Stewards, giving partial support to H3. Participants who believed more strongly that COVID-19 was a man-made, leaked biological weapon had higher odds of being Hygiene Stewards, Symptom Managers, and Refusers, in comparison to Adherent, but lower odds of being Social Distancers; giving partial support to H4.

Last we considered RQ2. In comparison to the Adherent class, participants with stronger levels of trait reactance had higher odds of being in all of the other classes. In contrast, participants who identified more strongly as health mavens had lower odds of being in all of the other classes.

\section{Exploring Predictors and Socio-demographics}

We explored whether the predictors varied by age, education, financial status, and gender. Some participants did not share this information, which reduced the sample size for some tests. Due to the number of tests, the threshold for statistical significance was increased to $p<.01$ for this subgroup analysis. Some of the socio-demographic variables were correlated. Perceived social status was positively associated with age, $r(936)=.33, p<.001$, and education, $r(963)=$ $.25, p<.001$. In addition, men reported higher perceived social status $(M=7.26, S D=1.52, n=$ 301 ) in comparison to women and non-binary individuals (collapsed into one group; $M=6.94$, $S D=1.48, n=665), t(964)=3.08, p<.01$.

Correlations showed that age was negatively associated with experiences of more negative than positive injunctive norms, $r(939)=-.22, p<.001$. In other words, the older participants were more likely to experience positive rather than negative injunctive norms. Older 
age was also associated with more household members living with chronic disease, $r(939)=.24$, $p<.001$, but fewer household members in essential worker positions, $r(939)=-.14, p<.001$.

Education was negatively associated with experiences of more negative than positive injunctive norms, $r(966)=-.12, p<.001$. In other words, participants with more education were more likely to experience positive rather than negative injunctive norms. Education was also negatively associated with belief that COVID-19 is a biological weapon, $r(964)=-.22, p<.001$.

Perceived social status was negatively associated with experiences of more negative than positive injunctive norms, $r(966)=-.14, p<.001$. In other words, participants with higher perceived social status were more likely to experience positive rather than negative injunctive norms. Higher perceived social status was also associated with fewer household members in essential worker positions, $r(966)=-.11, p<.001$.

Independent sample $t$ tests showed one difference by gender. Men showed higher levels of trait reactance $(M=2.51, S D=0.64, n=302)$ than women and non-binary individuals (collapsed into one group; $M=2.39, S D=0.69, n=668), t(968)=2.69, p<.01$.

\section{Discussion}

As we craft this paper, COVID-19 has claimed over 280,000 lives in the United States. Although there is promising news on vaccine development (Callaway, 2020), at this point, the best defense is mitigation. As COVID-19 infection rates rise (CDC, 2020c), and half of Americans do not plan to take a COVID-19 vaccine (Hamel et al., 2020), the need for thoughtfully planned communication campaigns has become urgent. Creating campaigns to slow the incidence and spread of COVID-19 is challenging because the goal—mitigationencompasses multiple behaviors. This study provided a novel approach to audience segmentation for a multifaceted goal, by using the person-centered approach of LCA and health 
communication theories to identify predictors. Five classes of mitigation were identified: one marked by complete adherence with health recommendations, one by complete refusal, and three by a mixture of adherence and refusal. These results reflect earlier findings that people may exhibit complex patterns of compliance and noncompliance (e.g., Bochner \& Insko, 1966; Smith et al., 2018).

\section{Adhering to COVID-19 Mitigation}

The descriptive statistics showed that a nontrivial number of participants did not intend to engage in recommended COVID-19 mitigation. Only half intended to maintain six feet of physical distance from others or to wash their hands. The LCA identified five classes based on their COVID-19 mitigation intentions, labeled as Adherents, Social Distancers, Hygiene Stewards, Symptom Managers, and Refusers. These results, on their own, provide a more refined way to segment a heterogeneous community into subgroups based on their pattern of responses than simply dividing audiences into complaint versus non-complaint or looking at intended compliance only through traditional social determinants (Block et al., 2020; Lennon et al., 2020). The classes also suggest at least three different types of campaign goals: to encourage Adherents to follow through on their intentions, to encourage Social Distancers and Hygiene Stewards to add one or two actions to their intentions, and to influence Symptom Managers to engage in infection prevention instead of just reacting to symptoms.

The Social Distancer and Hygiene Steward classes provide interesting insights into adoption. The common core across the three mixed classes (Social Distancers, Hygiene Stewards and Symptom Managers) was reacting to symptoms: covering faces when coughing or sneezing, staying home when sick, and seeking medical attention. Both Social Distancers and Hygiene Stewards added on one behavior that was novel for most of the U.S. public: wearing a face mask. 
In addition to masks, Social Distancers also intended to avoid crowds and maybe to maintain six feet of distance; Hygiene Stewards intended to wash their hands and (maybe) to avoid touching their faces with unwashed hands. Instead of a progression model of behavioral adoption, the classes reflect more of a fork in the road: for those who added prevention behaviors, after adding the face mask, they turned either to personal acts (handwashing, face touching) or to social ones (avoiding crowds and keeping physical distance). To join the Adherents, each class needs to take on new, but different, health actions. Effective campaigns may transition Hygiene Stewards directly to Adherent, without ever going through a Social Distancer phase (and vice versa). Both classes are about the same size (23-24\% of the sample); each could make a sizable improvement to the rather small Adherent class (34\%). The question for campaign creators is priorities.

It is unknown how difficult it would be to move people into classes with greater adherence. . Further research may elucidate answers to these questions and inform us about the sustainability of these potential changes (Hornik \& Woolf, 1999). However, since small movements in compliance yield large gains in disease mitigation (in nationally representative data, a 3\% increase in compliance is correlated with $9 \%$ fewer cases over the subsequent 30 days; Lennon et al., under review), approaches that target lowest-compliance behaviors in classes showing a willingness to adhere to some behaviors may be most effective. It is reasonable to think that it would be easier to move Symptom Managers to high compliance than to move those in the negligible compliance category of Refusers to high compliance. Table 3 enables messaging campaigns to be built around identifying a behavior (left column) and selecting a target audience (classes with low scores in the given behavior). For example, a hand-washing message will be more beneficial for Social Distancers and Symptom Managers than Hygiene Stewards. Alternatively, Table 3 enables campaigns to target audiences (right five columns) that focus on 
that class' specific needs (lowest behavior scores for a given class). For example, Hygiene Stewards would benefit from messages geared to physical distancing, avoiding touching face, and avoiding crowds.

Other considerations of movability include accommodating individual limitations in behaviors. For example, for persons who must commute to work on foot through crowds, an admonishment to avoid crowds will be of little value; however, a recommendation of strict adherence to mask wearing, avoiding touching face, and handwashing may help them overcome their (necessary) increased risk.

\section{Developing Targeted Campaigns}

The covariate analysis provided theoretical insight into possible strategies to pursue in campaigns targeting the four problematic classes. One finding was anticipated by the hazard outrage model (Sandman, 1993): outrage (i.e., the social/cultural aspects of risk), specifically the belief that COVID-19 was a man-made, leaked, biological weapon, was a strong predictor of class membership. Furthermore, stronger bioweapon beliefs predicted being Hygiene Stewards, Symptom Managers, and Refusers, rather than Adherents. In fact, more hazard-based risks (i.e., more essential workers in the household, more household members with chronic disease, and personally knowing someone who became sick with COVID-19) often predicted being in a problematic class, rather than Adherent. This finding would not have surprised Sandman (1993), but it does contradict the intense attention in risk and health communication on hazard-type risks and suggests campaign designers need to put greater focus on outrage-type risks.

Additionally, injunctive norms were predictive of every problematic classes (with minimal effects for Social Distancers): as people reported more experiences of negative rather than positive injunctive norms, they were not likely to be Adherents. As anticipated by work on 
conformity (Cialdini \& Goldstein, 2004) and theories of normative influence (e.g., TNSB, Rimal \& Real, 2005), people may experience approval or disapproval from the important people in their lives. As researchers, we need to avoid a positivity bias in our consideration of injunctive norms. Disapproval is powerful: even watching someone else face disapproval shapes behavior (Cialdini \& Goldstein, 2004; see also social cognitive theory's [Bandura, 2001] predictions for observational learning). "Taken together, these results suggest that even when not directly, personally, or publicly the target of others' disapproval, individuals may be driven to conform to restore their sense of belonging and their self-esteem" (p. 611).

Although we have guidance on how to convey descriptive norms (typical behavior), less attention has been paid to constructing messages that adjust injunctive norms. As recommended by Goldberg et al. (2020): "[the] proportion of Americans performing preventive behaviors will substantially increase if people communicate to friends and family that they are engaging in these behaviors themselves, and that you should too, to keep yourself and others safe" (p. 5). It may be more challenging to overcome negative injunctive norms. In our sample, those facing more negative injunctive norms were younger, less educated, with a lower financial status. This finding, then, provides an alternative explanation for lower compliance among younger adults: they may not be apathetic or need to be frightened (Noar \& Austin, 2020), they may be experiencing teasing, harassment, and social exclusion for their actions at a time when such social connections and affiliation is deeply meaningful. It is possible that a positive-injunctive initiative may counter-balance the experiences of those facing more negative injunctive norms.

Targeting injunctive norms and bioweapon beliefs could be a powerful approach for reaching Hygiene Stewards, Symptom Managers, and Refusers. An important finding from the covariate analysis is the lack of prediction around Social Distancers. Across the board, the odds 
ratios and beta estimates were very small for this class. The predictors revealed more about why someone would be Adherent (vs. Hygiene Stewards, Symptom Managers, and Refusers), but not a Social Distancer. This finding suggests that there is much for us to learn about why people adopt physical distancing (interpersonally or crowds) instead of washing their hands and avoiding touching their faces with unwashed hands.

The covariate analysis showed that people with stronger health mavenism had much lower odds of being in a problematic class versus being Adherents. This opinion leader trait describes people who like to be sources for health expertise and advice (Boster et al., 2011) and the good news is that they are more likely to intend to engage in the recommended COVID-19 mitigation. Then again, their lack of appearance in the other classes makes them less useful targets as agents of change in the other classes; they may be seen as outsiders. Thus, the best use of health mavens may be to bolster the intentions of Adherents, helping them to follow through on their intentions. This analysis also revealed that those high in trait reactance had higher odds of being in all of the other classes than being Adherents. As such, messages trying to move individuals toward Adherents may want to avoid freedom-restricting language that could foster reactance.

\section{Limitations}

This sample provides insights into the lived experiences of a community living through the pandemic together, but due to the small sample size and lack of diversity, population-level studies should assess how well these results generalize to the U.S. population. In addition, while self-reported intentions are useful for formative research and planning, there is not a perfect relationship between intentions and self-reported behavior (e.g., Sheppard, Hartwick, \& Warshaw, 1988) or observed behavior. 
The data also represent one point in time in a pandemic. Behavioral adoption and maintenance, are expected to be dynamic, even without intervention. As seen in studies of diffusion of innovations (Rogers, 2003), it may be too exhausting to maintain, or even intend to maintain, all mitigation behaviors for long periods of time. On the other hand, as some novel behaviors become easier and habitual, people may be able to take on more. Tracking this cohort over time would allow us insight into the transitions between classes and the predictors of transitions, such as exhaustion or disenchantment.

\section{Conclusion}

Given the multiple behaviors needed to ensure adequate COVID-19 mitigation, designing an effective campaign requires sophisticated audience segmentation. By taking a person-centered approach and applying relevant theoretical principles, the present study offers campaign designers theoretically and empirically informed insights. Although further research is needed to fully parse the relative movability and sustainability of movements by behavior and by class, there are several clear candidates for targeted messaging as we attempt to address the most pressing infectious disease in a century. 


\section{References}

Adler, N. E., \& Stewart, J. (2007, March). The MacArthur Scale of Subjective Social Status. Retrieved from https://macses.ucsf.edu/research/psychosocial/subjective.php

Alsan, M,, Stantcheva, S, Yang, D., \& Cutler, D. (2020). Disparities in Coronavirus 2019 reported incidence, knowledge, and behavior among US adults. JAMA Network Open, 3(6): e2012403, 1-11. doi:10.1001/jamanetworkopen.2020.12403

Bandura, A. (2001). Social cognitive theory: An agentic perspective. Annual Review of Psychology, 52, 1-26. https://doi.org/10.1146/annurev.psych.52.1.1

Becker, M. H. (1974). The health belief model and sick role behaviour. Health Education Monographs, 2, 409-419. https://doi.org/10.1177/109019817400200407

Block, R., Berg, A., Lennon, R. P., Miller, E. L., \& Nunez-Smith, M. (2020). African American adherence to COVID-19 public health recommendations. Health Literacy Research and Practice, 4(3), e166-e170.

Bochner, S., \& Insko, C. A. (1966). Communicator discrepancy, source credibility, and opinion change. Journal of Personality and Social Psychology, 4, 614-621. doi: $10.1037 / \mathrm{h} 0021192$

Boster, F. J., Kotowski, M. R., Andrews, K. R., \& Serota, K. (2011). Identifying influence: Development and validation of the connectivity, persuasiveness, and maven scales. Journal of Communication, 61, 178-196. doi:10.1111/j.1460-2466.2010.01531.x

Brehm, S. S., \& Brehm, J. W. (1981). Psychological reactance: A theory of freedom and control. New York, NY: Academic Press.

Burgoon, M., Alvaro, E., Grandpre, J., \& Voloudakis, M. (2002). Revisiting the theory of psychological reactance: Communicating threats to attitudinal freedom. In J. Dillard \&M. 
Pfau (Eds.), The persuasion handbook: Theory and practice (pp. 213-232). Thousand Oaks, CA: Sage.

Callaway, E. (2020, November 16). COVID vaccine excitement builds as Moderna reports third positive result. Nature, 587, 337-338. https://doi.org/10.1038/d41586-020-03248-7

Centers for Disease Control and Prevention. (2020a, November 4) Coronavirus disease 2019 (COVID-19): How to protect yourself \& others. https://www.cdc.gov/coronavirus/2019ncov/prevent-getting-sick/prevention.html

Centers for Disease Control and Prevention. (2020b, November 2). Coronavirus Disease (COVID-19): Evidence used to update the list of underlying medical conditions that increase a person's risk of severe illness from COVID-19. https://www.cdc.gov/coronavirus/2019-ncov/need-extra-precautions/evidence-table.html Centers for Disease Control and Prevention (2020c, November 13). Coronavirus Disease 2019 (COVID-19): National COVID-19 Activity Indicators. https://www.cdc.gov/coronavirus/2019-ncov/covid-data/covidview/index.html

Cialdini, R. B., \& Goldstein, N. J. (2004). Social influence: Compliance and conformity. Annual Review of Psychology, 55, 591-621. doi:10.1146/annurev.psych.55.090902.142015.

Collins, L. M., \& Lanza, S. T. (2010). Latent class and latent transition analysis: With applications in the social, behavioral, and health sciences. New York, NY: Wiley.

Cucinotta, D. \& Vanelli, M. (2020) WHO Declares COVID-19 a Pandemic. Acta Biomedica, 91(1), 157-160. doi: 10.23750/abm.v91i1.9397

Dillard, J. P., \& Shen, L. (2005). On the nature of reactance and its role in persuasive health communication. Communication Monographs, 72(2), 144-168. doi:10.1080/03637750500111815 
Fishbein, M., \& Ajzen, I. (2010). Predicting and changing behavior: The reasoned action approach. New York, NY: Psychology Press.

Goldberg, M. H., Gustafson, A., Maibach, E. W., van der Linden, S., Ballew, M. T., Bergquist, P., Kotcher, J. E., Marlon, J.R., Rosenthal, S. A., \& Leiserowitz, A. (2020). Social norms motivate COVID-19 preventive behaviours. PsyArXiv. 2020.

Gover, A. R., Harper, S. B., \& Langton, L. (2020). Anti-Asian hate crime during the COVID-19 pandemic: exploring the reproduction of inequality. American Journal of Criminal Justice, 45, 647-667. https://doi.org/10.1007/s12103-020-09545-1

Hong, S. M., \& Faedda, S. (1996). Refinement of the Hong Psychological Reactance Scale. Educational and Psychological Measurement, 56, 173-182. doi: $10.1177 / 0013164496056001014$

Hamel, L., Kearney, A., Kirzinger, A., Lopes, L., Muñana, C., \& Brodie, M. (2020). Top issues in 2020 election, the role of misinformation, and views on a potential Coronavirus vaccine. https://www.kff.org/coronavirus-covid-19/report/kff-health-tracking-pollseptember-2020/

Hornik, R., \& Woolf, K. D. (1999). Using cross-sectional surveys to plan message strategies. Social Marketing Quarterly, 5, 34-41. doi: 10.1080=15245004.1999.9961044

Kreuter, M., Farrell, D., Olevitch, L., \& Brennan, L. (2000). Tailoring health messages: Customizing communication with computer technology. Mahwah, NJ: Lawrence Erlbaum.

Lanza, S. T., Collins, L. M., Lemmon, D. R., \& Schafer, J. L. (2007). PROC LCA: A SAS procedure for latent class analysis. Structural Equation Modeling, 14, 671-694. doi:10.1080/10705510701575602 
Lennon, R. P., Sakya, S., Miller, E. L., Snyder, B., Yaman, T., Zgierska, A. E., Ruffin, M. T., \& Van Scoy, L. J. (2020). Public intent to comply with COVID-19 public health recommendations. Health Literacy Research and Practice, 4(3): e161-e165.

Lennon, R. P., Zgierska, A. E., Miller, E. L., Snyder, B., Keshaviah, A., Hu, X. C., Zhou, H., \& Van Scoy, L.J. (under review). Lower intent to comply with COVID-19 public health recommendations correlates to higher disease burden of the following 30 days. American Journal of Public Health.

Maibach, E. W., Leiserowitz, A., Roser-Renouf, C., \& Mertz, C. K. (2011). Identifying likeminded audiences for global warming public engagement campaigns: An audience segmentation analysis and tool development. PloS One, 6(3), 1-9. doi:10.1371/journal.pone.0017571

Maibach, E., Maxfield, A., Ladin, K., \& Slater, M. (1996). Translating health psychology into effective health communication: The American Health styles Audience Segmentation Project. Journal of Health Psychology, 1, 261-277. doi: 10.1177/135910539600100302

McPherson, M., Smith-Lovin, L., \&Cook, J. M. (2001). Birds of a feather: Homophily in social networks. Annual review of sociology, 27 (1), 415-444. https://www.annualreviews.org/doi/abs/10.1146/annurev.soc.27.1.415

Montgomery, G. H., Erblich, ., DiLorenzo, T., \& Bovbjerg, D. H. (2003). Family and friends with disease: Their impact on perceived risk. Preventive Medicine, 37 (3), 242-249. https://doi.org/10.1016/S0091-7435(03)00120-8

Noar, S. M., \& Austin, L. (2020, online first). (Mis)communicating about COVID-19: Insights from Health and Crisis Communication. Health Communication, 1-5. doi:10.1080/10410236.2020.1838093 
Noar, S. M., Benac, C. N., \& Harris, M. S. (2007). Does tailoring matter? Meta-analytic review of print health behavior change interventions. Psychology Bulletin, 133(4), 673-693. doi:10.1037/0033-2909.133.4.673

Noar, S. M., Harrington, N. G., \& Aldrich, R. S. (2009). The role of message tailoring in the development of persuasive health communication messages. Communication Yearbook, $33,73-133$.

Park, H. S., \& Smith, S. (2007). Distinctiveness and influence of subjective norms, personal and descriptive injunctive norms, and societal descriptive and injunctive norms on behavioral intent: A case of two behaviors critical to organ donation. Human Communication Research, 33, 194-218. doi:10.1111/j.1468-2958.2007.00296.x.

Reno, R., Cialdini, R., \& Kallgren, C.A. (1993). The transsituational influence of social norms. Journal of Personality and Social Psychology, 64, 104-112.

Rimal, R. N., \& Lapinski, M. K. (2015). A re-explication of social norms, ten years later. Communication Theory, 25, 393-409. doi:10.1111/comt.12080

Rimal, R. N., \& Real, K. (2005). How behaviors are influenced by perceived norms: A test of the theory of normative social behavior. Communication Research, 32, 389-414. doi:10.1177/0093650205275385.

Rogers, E. M. (2003). Diffusion of innovations. New York, NY: Simon \& Schuster.

Rogers, R. W. (1975). A protection motivation theory of fear appeals and attitude change. Journal of Psychology, 91, 93-114.

Sandman, P. M. (1993). Community outrage: Strategies for effective risk communication. Fairfax, VA: American Industrial Hygiene Association.

Sheeran, P., Maki, A., Montanaro, E., Avishai-Yitshak, A., Bryan, A., Klein, W. M., ... \& 
Rothman, A. J. (2016). The impact of changing attitudes, norms, and self-efficacy on behavior: a meta-analysis. Health Psychology, 35(11), 1178-1188.4.

Shen, L., \& Dillard, J. P. (2005). Psychometric properties of the Hong psychological reactance scale. Journal of Personality Assessment, 85(1), 74-81. doi:10.1207/s15327752jpa8501_07

Sheppard, B. H., Hartwick, J., \& Warshaw, P. R. (1988). The theory of reasoned action: A metaanalysis of past research with recommendations for modifications and future research. Journal of Consumer Research, 15, 325-343. doi: 10.1086/209170

Slater, M. D. (1996). Theory and method in health audience segmentation. Journal of Health Communication: International Perspectives, 1, 267-284. doi: 10.1080/108107396128059

Slater, M. D., \& Flora, J. A. (1991). Health lifestyles: Audience segmentation analysis for public health interventions. Health Education \& Behavior, 18, 221-233.

Slater, M. D., Kelly, K. J., \& Thackeray, R. (2006). Segmentation on a shoestring: Health audience segmentation in limited-budget and local social marketing interventions. Health Promotion Practice, 7(2), 170-173. doi:10.1177/1524839906286616

Smith, R. A. (2017). Audience segmentation techniques. In R. L. Parrott (Ed.), Oxford research encyclopedia of communication: Health and risk message design and processing (pp. 112). New York, NY: Oxford University Press. https://doi.org/10.1093/acrefore/9780190228613.013.321

Smith, R. A., Kim, Y. L., \& M'ikantha, N. M. (2018). Identifying and predicting profiles of medical noncompliance: Pediatric caregivers' antibiotic stewardship. Journal of Health Communication, 23, 485-494. doi: 10.1080/10810730.2018.1471109.

Storey, J. D., Saffitz, G. B., \& Rimon, J. G. (2008). Social marketing. In K. Glanz, B. K. Rimer, 
\& K. Viswanath (Eds.), Health Behavior and Health Education: Theory, Research, and Practice (pp. 435-446). San Francisco, CA: Jossey-Bass.

Van Scoy, L. J., Miller, E. L., Snyder, B., Wasserman, E., Chincilli, V. M., Zgierska, A. E., Rabago, D., Lennon, C. L., Lipnick, D., Toyobo, O., Ruffin, M. T., \& Lennon, R. P. (in press). Knowledge, perceptions, and preferred information sources related to COVID-19 among central Pennsylvania adults early in the pandemic: results of a mixed methods cross sectional survey. Annuals of Family Medicine.

Witte, K. (1994). Fear control and danger control: A test of the extended parallel process model. Communication Monographs, 61, 113-134. doi:10.1080/03637759409376328 
Table 1

Intentions to Engage in COVID-19 Mitigation: Indicators for Latent Class Analysis ( $\mathrm{N}=976)$

\begin{tabular}{lcccc}
\hline Indicators & Code & Label & $n$ & $\%$ \\
\hline Wash hands often with soap & 1 & Never to Sometimes & 467 & 47.8 \\
& 2 & Always & 509 & 52.2 \\
Wear a face mask when out in public & 1 & Never to Sometimes & 222 & 22.7 \\
& 2 & Always & 754 & 77.3 \\
Avoid touching face with unwashed hands & 1 & Never to Sometimes & 582 & 59.6 \\
& 2 & Always & 394 & 40.4 \\
Cover mouth/nose when cough or sneeze & 1 & Never to Sometimes & 190 & 19.5 \\
& 2 & Always & 786 & 80.5 \\
Stay home if feel sick & 1 & Never to Sometimes & 136 & 13.9 \\
Stay 6 feet away from others in public & 2 & Always & 840 & 86.1
\end{tabular}


Table 2

Model Fit Information for Comparison of Latent Class Models

\begin{tabular}{crrrr}
\hline Number of classes & $\mathrm{G}^{2}$ & AIC & BIC & $d f$ \\
\hline 2 & 672.03 & 706.03 & 789.05 & 238 \\
3 & 338.21 & 390.21 & 517.18 & 229 \\
4 & 269.76 & 339.76 & 510.68 & 220 \\
$\mathbf{5}$ & $\mathbf{2 0 0 . 4 1}$ & $\mathbf{2 8 8 . 4 1}$ & $\mathbf{5 0 3 . 2 9}$ & $\mathbf{2 1 1}$ \\
7 & 159.40 & 265.40 & 524.22 & 202 \\
\hline
\end{tabular}

Note. Boldface type indicates the selected model. AIC=Akaike's information criterion; $\mathrm{BIC}=$ Bayesian information criterion; $d f=$ degrees of freedom . 
Table 3

Item-Response Probabilities for Five-Class Model Given Latent Class Membership

\begin{tabular}{lccccc}
\hline & Adherents & $\begin{array}{c}\text { Social } \\
\text { Distancers }\end{array}$ & $\begin{array}{c}\text { Hygiene } \\
\text { Stewards }\end{array}$ & $\begin{array}{c}\text { Symptom } \\
\text { Managers }\end{array}$ & Refusers \\
& $34 \%$ & $24 \%$ & $23 \%$ & $10 \%$ & $9 \%$ \\
\hline Wash hands & $\mathbf{. 9 3}$ & .13 & $\mathbf{. 6 9}$ & .02 & .19 \\
Wear face mask & $\mathbf{9 7}$ & $\mathbf{. 8 6}$ & $\mathbf{. 8 2}$ & .34 & .17 \\
Avoid touching face & $\mathbf{. 8 4}$ & .09 & .42 & .02 & .01 \\
Cover sneeze/cough & $\mathbf{. 9 9}$ & $\mathbf{. 7 4}$ & $\mathbf{. 9 3}$ & $\mathbf{. 5 7}$ & .23 \\
Stay home if ill & $\mathbf{1 . 0 0}$ & $\mathbf{. 9 2}$ & $\mathbf{. 9 0}$ & $\mathbf{. 9 0}$ & .06 \\
Seek medical & $\mathbf{. 9 7}$ & $\mathbf{. 8 7}$ & $\mathbf{. 8 4}$ & $\mathbf{. 9 2}$ & .11 \\
Physical distance & $\mathbf{. 9 4}$ & $\mathbf{. 5 1}$ & .23 & .00 & .00 \\
Avoid crowds & $\mathbf{9 4}$ & $\mathbf{. 8 9}$ & .44 & .24 & .00 \\
\hline
\end{tabular}

Note. Percentages reflect the number of participants likely to be in each class. Cells contain the likelihood of "always" doing the COVID-19 related behavior. Likelihoods over 50\% appear in bold. 
Table 4

Covariate Analysis with Adherents as the Referent Class

\begin{tabular}{|c|c|c|c|c|c|c|c|c|c|}
\hline & \multicolumn{2}{|c|}{ Social Distancers } & \multicolumn{2}{|c|}{ Hygiene Stewards } & \multicolumn{2}{|c|}{ Symptom Managers } & \multicolumn{2}{|c|}{ Refusers } & \multirow[b]{2}{*}{$\mathrm{LL}^{2}$} \\
\hline & OR & $\begin{array}{c}B \\
(S E)\end{array}$ & $O R$ & $\begin{array}{c}B \\
(S E)\end{array}$ & $O R$ & $\begin{array}{c}B \\
(S E)\end{array}$ & $O R$ & $\begin{array}{c}B \\
(S E) \\
\end{array}$ & \\
\hline Relative: Neg. injunctive norms & 1.02 & $\begin{array}{c}0.02 \\
(0.07)\end{array}$ & 1.34 & $\begin{array}{c}0.29 \\
(0.07)\end{array}$ & 1.36 & $\begin{array}{c}0.31 \\
(0.08)\end{array}$ & 1.41 & $\begin{array}{c}0.35 \\
(0.08)\end{array}$ & $22.31 * *$ \\
\hline Essential worker risk & 0.89 & $\begin{array}{l}-0.12 \\
(0.11)\end{array}$ & 1.38 & $\begin{array}{c}0.32 \\
(0.11)\end{array}$ & 1.27 & $\begin{array}{c}0.24 \\
(0.13)\end{array}$ & 1.59 & $\begin{array}{c}0.46 \\
(0.12)\end{array}$ & $14.83 * *$ \\
\hline Know COVID-19 patient & 0.92 & $\begin{array}{l}-0.08 \\
(0.13)\end{array}$ & 0.81 & $\begin{array}{l}-0.22 \\
(0.16)\end{array}$ & 1.60 & $\begin{array}{c}0.47 \\
(0.20)\end{array}$ & 0.58 & $\begin{array}{l}-0.55 \\
(0.19)\end{array}$ & $10.83 *$ \\
\hline Biological weapon belief & 0.89 & $\begin{array}{l}-0.12 \\
(0.08)\end{array}$ & 1.22 & $\begin{array}{c}0.20 \\
(0.08)\end{array}$ & 1.35 & $\begin{array}{c}0.30 \\
(0.09)\end{array}$ & 1.35 & $\begin{array}{c}0.30 \\
(0.09)\end{array}$ & $21.00^{* *}$ \\
\hline
\end{tabular}

Note. $O R=$ Odds ratio; $B=$ unstandardized beta estimate; $S E=$ standard errors. $L L=$ Log-likelihood. Relative: Neg. Injunctive norm $=$ experiences of negative injunctive norms - experiences of positive injunctive norms.

$* p<.05 ; * * p<.01 ; * * * p<.001$ 